\title{
The use of pneumatic cylinders with a return spring to compensate for balance losses in mechanical regenerative drives for reciprocating movements
}

\author{
Wen Zhao ${ }^{1, *}$ and Victor Leonidovich Zhavner $^{1}$ \\ ${ }^{1}$ Peter the Great St. Petersburg Polytechnic University, Automat Department, 195221 Politechnicheskaya St. 29, Russian Federation
}

\begin{abstract}
Mechanical spring drives with energy recovery are designed to produce return-rotational movements in various technological equipment and reduce energy costs by several times compared with traditional electromechanical, hydraulic and pneumatic drives. The use of pneumatic motors in spring drives to compensate for dissipative losses, fixing the drive in extreme positions and the primary charging of spring batteries is considered. The class of tasks in which spring drives with energy recovery are created using only pneumatic cylinders with return springs is highlighted. This study examines mechanical spring drives with energy recovery, based on nonlinear spring batteries with spring preload in the middle position of the output link. The use of pneumatic actuators with return springs in mechanical drives based on linear spring batteries with two springs is proposed. Mechanical spring drives with energy recovery are designed to produce return-rotational movements in various technological equipment and reduce energy costs by several times compared with traditional electromechanical, hydraulic and pneumatic drives. The amount of energy expended in spring recuperative drives is actually determined by the energy expended on compensating for dissipative losses in kinematic pairs. The results of the study allow the designers of such drives to consciously approach the choice of their schemes and design parameters.
\end{abstract}

\section{Introduction}

Traditionally, spring drives are used in process equipment to perform work operations that require high speed, while they work in pairs and in series with another drive, usually electromagnetic, providing the accumulation of potential energy in the spring [1]. The history of spring drives is primarily associated with the development of a mechanical clock, using a spiral spring as a drive $[2,3]$. The clock is an example of a selfoscillatory system, the issues of compensating dissipative losses were solved by the Dutch mathematician Erie [4, 5]. The essence of the Erie theorem is as follows: the supply of a compensation impulse at the beginning of the movement reduces the oscillation period, the supply of the compensation impulse in the middle of the stroke saves the oscillation period, and the supply of a pulse at the end of the stroke increases the oscillation period. Details are considered in the work of N. N. Bautin [5].

The next milestone in the development of spring drives was the invention of L. Szilard and A. Einstein of an electromagnetic device for obtaining oscillatory movements [6]. The device was intended for use in compressors of refrigeration machines and its scheme is shown in Fig. 1.
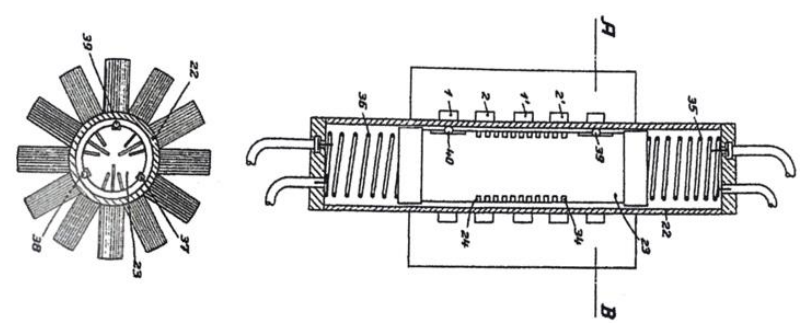

Fig. 1. Diagram of refrigeration compressors for Patent No. 562040 (Germany)

In this embodiment, the piston of the refrigeration machine is located between the two compression springs and the operation of the spring drive and the linear electromagnetic drive occurs simultaneously. The spring drive performs acceleration in this case, the electromagnetic linear drive is activated, under the action of which the refrigerant and the second spring are compressed. After the piston stops, the force of the linear electromagnetic drive is zero and the movement in the opposite direction begins under the action of a compressed spring. It should be noted that the explanation of the principle of operation of the device in the patent is given by the example of the returnrotational movement of the output link connected to the spiral springs [6]. At that time, the Swedish company Electrolux was engaged in refrigeration and, according to "Einstein inventor" [7], some patents by L. Szilard

\footnotetext{
*Corresponding author: wenfly2015@yandex.ru
} 
and A. Einstein were acquired by this company. In 1975, Electrolux received a patent for a device in which the output link performs a reciprocating rotational movement under the action of a spiral spring with a linear torque characteristic, the drive is also equipped with two electromagnets and two position sensors connected to the control system [8]. It was a mechanical spring drive with energy recovery, where the turning time was determined by the spring stiffness and the moment of inertia of the output link. Electromagnets in this drive perform the function of clamps and provide compensation for dissipative losses in the support and from internal friction in the spring. The compensation impulse can be given: at the beginning of the movement, at the end of the movement, at the beginning and the end of the movement.

This spring drive is a self-oscillating system with energy pumping from the outside and with controlled standoff provided by clamps, position sensors and a control system.

The demonstration of the mechanical arm at a conference in Moscow in 1980 attracted the attention of the scientific and technical community to this subject and here it is necessary to single out the first patents [9, 10]. In the first case, during the technological pause, the potential energy of the spring accumulator is increased, which ensures the law of motion close to the cosine. The limitation to the use of this method of compensating dissipative losses is an increase in engine power, with short technological breaks in time. In the second patent [10], a device for stepping rotational movements with a spring accumulator in the form of a helical spring was proposed.

Theoretical issues related to the ideology of designing spring drives with energy recovery are considered in [11-14]. Basically, in these works, drives were built using linear spring batteries and electric motors to compensate for dissipative losses. In the study of $[15,16]$, the properties of nonlinear spring accumulators based on helical coil springs were investigated. In the work [17], it is shown that in a nonlinear spring accumulator there is a special case when in the neutral position the spring has tension and at certain characteristics throughout the course of the carriage the load on the guides is zero, and the spring accumulator has the characteristics of a harmonic oscillator.

From the analysis of the published works, it can be concluded that mechanical spring drives with energy recovery have two significant advantages over conventional type-cycle drives: favorable dynamic conditions and a significant reduction in the cost of energy. In the work [18] it is shown that for a traditional cyclic drive with the law of motion with constant acceleration with increasing speed, for example, twice the energy consumption increases four times, and the required engine power eight times.

A comparative analysis of mechatronic spring drives with energy recovery, based on linear spring batteries, with electromechanical, pneumatic and hydraulic drives, was performed in the work [19]. It is shown that mechatronic spring drives have advantages over traditional drives not only in favorable dynamic characteristics, but also in a significant reduction in weight and size characteristics.

The advantage of mechatronic spring drives with energy recovery is: cost reduction by more than 5 times, favorable dynamic modes, they are well integrated into modern automated control systems, can be programmed in a single control system of technological equipment or have a specialized control system, reduced massdimensional characteristics of drives, insensitivity to changes in ambient temperature.

\section{Object of Study}

The object of the study is a nonlinear spring accumulator based on a rocker mechanism with one compression spring, the system of which is shown in Fig. 2. The general case is considered when the carriage has a predeformation of the spring $s_{l}$ in the neutral position.

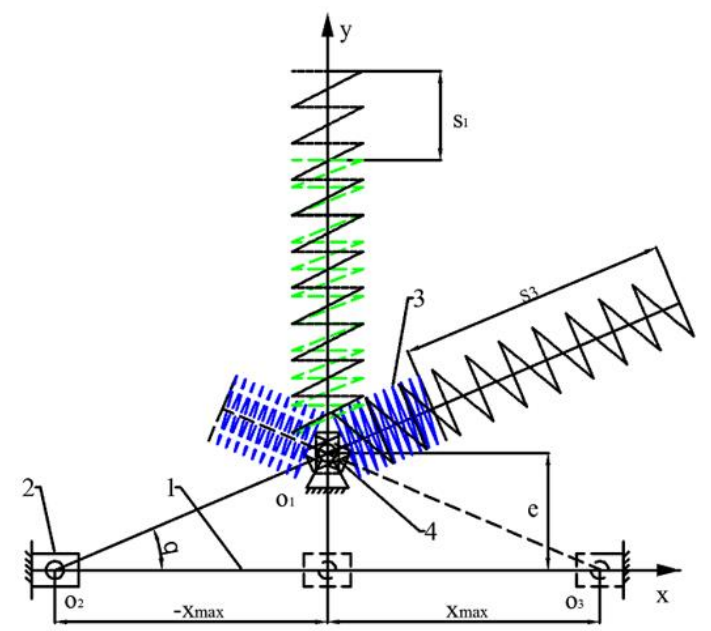

Fig. 2. System of a nonlinear spring accumulator with one compression spring.

The preliminary deformation of the spring lies within $s_{1}=0-2 e$.

If according to the operation [17], the spring stiffness is $c=m g / \mathrm{e}$, then the preliminary spring force is $F_{l}=c s_{1}$.

Fig. 3. shows the bending lines with extreme values of $s_{l}$, when passing the carriage of the middle position. When $F_{l}=m g$ in this position, the deflection is zero.
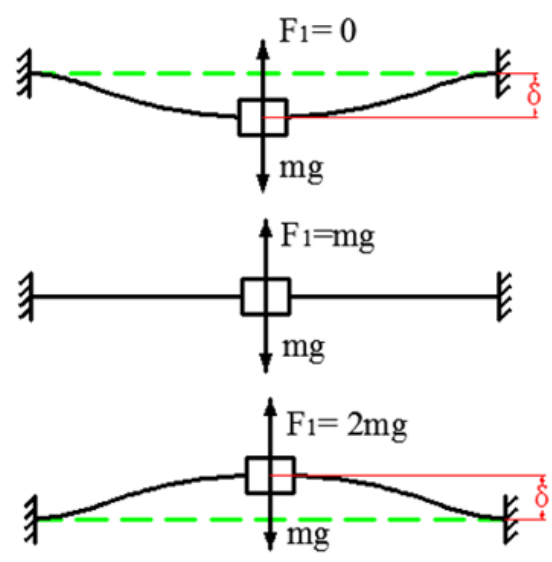

Fig. 3. Bend lines for $s_{1}=0, s_{l}=e$ and $s_{I}=2 e$. 
The purpose of the study is to determine the main characteristics of the spring battery with given characteristics of the working operations:

- $m$ the moving mass,

- $S$ the distance between the extreme positions,

- $t$ the move time.

In this paper, we consider working operations in which the maximum values of the reduced masses $m$ are in the range of up to $6000-8000 \mathrm{~kg}$, the maximum displacement $S$ is up to 2 meters $(2000 \mathrm{~mm})$, and the move time is considered within the range of up to $2 \mathrm{~s}$.

The essential features of such a drive:

1. The dimension in the direction of the carriage movement slightly exceeds the amount of displacement $S$.

2. Dissipative losses due to friction forces appear in the hinged joints and in translation pairs of the rocker mechanism, depending on the spring force.

The geometric characteristics of the spring battery on the basis of the rocker mechanism are:

1. $x_{\max }$ - the maximum carriage offset from the neutral position;

$$
x_{\max }=S / 2,
$$

2. $e$ - the distance along the normal between the axis of attachment of the rocker stone to the base to the line of movement of the carriage;

3. $s_{1}-$ preliminary deformation of the spring;

4. $s_{3}$ - maximum deformation of the spring;

5. $h$ - the maximum stroke of the spring, $h=s_{3^{-}} s_{1}$.

Other characteristics of the spring accumulator are:

$U(x)$ - the law of change of potential energy $U$ depending on the magnitude of the carriage movement $x$;

$t$ - time of carriage movement between its extreme positions;

$F(x)$ - the dependence of the spring force $F$ on the magnitude of the movement of the carriage;

$c$ - spring stiffness;

$F_{\text {max }}$ - maximum spring force;

$F x(x)$ - the law of change of the driving force of the spring along the guides, which at a constant mass is similar to the law of acceleration;

$F y(x)$ - the law of change in the force of the spring acting on the guides along the normal to them;

$\dot{x}(x)$ - the dependence of the carriage speed on the magnitude of the carriage movement;

$A_{d}$ - the value of dissipative losses in the spring accumulator, which determines the energy consumption of the spring drive with energy recovery.

Knowledge of these characteristics allows the designer to consciously select their values and perform a comparative analysis of the drives at the initial design stage.

To establish the relationship between the above characteristics and parameters, we use the equation of conservation of mechanical energy of the considered oscillatory system without taking into account dissipative losses.

$$
T=U C E=\frac{m x^{2}}{2}
$$

where, $T$ is the current value of the kinetic energy of the system;

$E=0.5 c\left(h+s_{1}\right)^{2}$, maximum potential energy of the spring, and $h=\sqrt{x_{\max }^{2}+e^{2}}-e$-maximum working extension of the spring.

The current value of the potential energy is

$$
U=0.5 c\left(\sqrt{x^{2}+e^{2}}-e+s_{1}\right)^{2},
$$

The minimum value of potential energy is

$$
U_{\min }=0.5 c s_{1}^{2},
$$

With a preliminary spring force, the change in potential energy occurs within E-Umin-E. The characteristics of the spring accumulator also depend on the effective potential energy $U_{\ni}=E-U m i n$.

Travel time to distance $S$ is determined from the expression [15]

$$
t=\sqrt{2 m} \int_{0} \frac{d x}{\sqrt{E-U_{\text {min }}}}
$$

After the transformations of equation (5), taking into account equations (1-4), we obtain

$$
\mathrm{t}=K_{Q} \sqrt{m / c},
$$

Where, $K_{0}-$ dimensionless coefficient obtained by solving an integral equation.

$$
K_{0}=\int_{-1}^{1} \frac{d x}{\sqrt{\sqrt{\left(\sqrt{1+\epsilon^{2}}-6+E_{0}\right)^{2}-\left(\sqrt{x^{2}+\epsilon^{2}}-6+E_{D}\right)^{2}}}},
$$

where, $\bar{e}=e / x_{\max }, \bar{x}=x / x_{\max }, \bar{s}_{1}=s_{1} / x_{\max }$.

Fig. 4. shows the graphs of the change in the $K_{0}$ coefficient depending on the parameters $\bar{e}$ and $\bar{s}_{l}$.

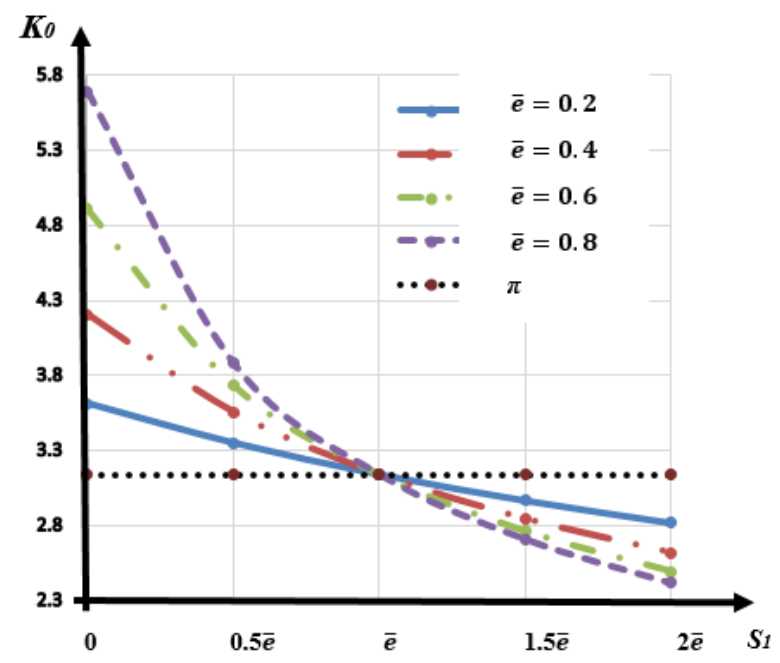

Fig. 4. Graphs of changes in $K_{0}$ coefficients.

From the graphs it can be seen that all the curves intersect at one point $e=s_{l}$, where the coefficient $K_{0}$ is equal to $\pi$. When $s_{1}$ is greater than $e$, the coefficient $K_{0}$ is less than $\pi$. Consequently, it is possible to change the time of movement to the lower side, increasing the preliminary tension of the spring $s_{l}$. 
The maximum spring force is:

$$
E_{\max }=c \cdot\left(\sqrt{1+\bar{e}^{2}}-\bar{e}+\bar{s}_{1}\right) \cdot x_{\max } .
$$

Fig. 5. shows the graphs of the change in the maximum spring force as a function of $\bar{e}$ and $\bar{s}_{\mathbb{l}}$ with a unit mass $m=I, x_{\max }=1$.

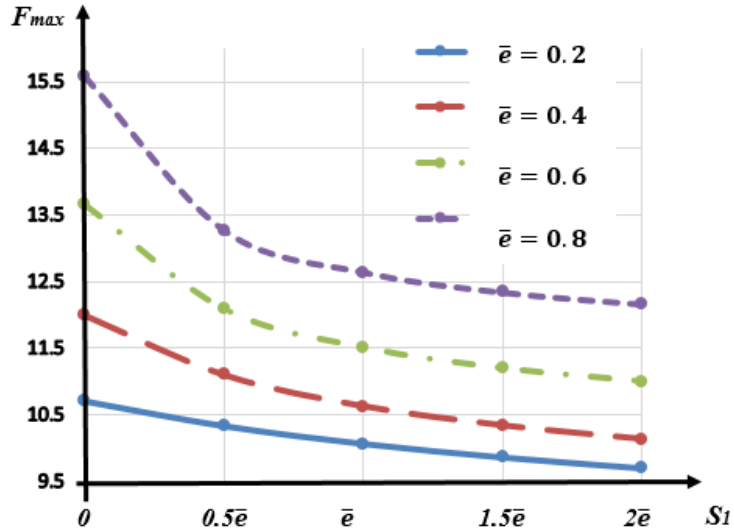

Fig. 5. Graphs of changes in the maximum spring force depending on the pretension.

The maximum value of the spring force is decisive when selecting a lock. For example, when using hydraulic or pneumatic cylinders mounted parallel to the springs, their force should be equal to the maximum spring force.

Note also that if the force of the pneumatic cylinder is equal to or greater than the spring force, then the carriage is automatically locked in the extreme positions.

The spring force along the carriage direction is equal to

$$
F_{x}=c \cdot\left(\sqrt{\bar{x}^{2}+\bar{\theta}^{2}}-\bar{\theta}+\bar{s}_{1}\right) \cdot x_{\max } \cdot \cos q .
$$

When $\bar{e}=\bar{s}_{\ell}$, the equation (9) takes the form $F_{x}=c x$, and the nonlinear spring accumulator acquires the properties of a linear harmonic oscillator.

Spring force acting normal to the direction of movement is determined from the expression

$$
F_{y}=c \cdot x_{\max } \cdot F_{y}^{v},
$$

where, $F_{y}^{v}=\bar{e} \cdot\left(1-\frac{\epsilon-s_{f}}{\sqrt{x^{2}+\epsilon^{2}}}\right)$.

Fig. 6. shows the graphs of $F_{y}^{v}$ along the guides when $\bar{s}_{1}=0, \bar{x}=-1 \ldots 0 \ldots 1$.

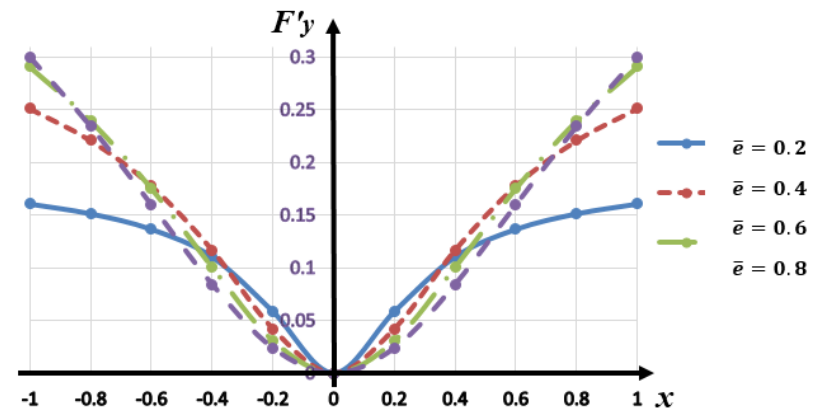

Fig. 6. Graphs of $\bar{F}_{y}^{x}$ along the guides when $\bar{s}_{1}=0$.
Fig. 7. shows the graphs of $F_{y}^{v}$ along the guides when $\bar{s}_{1}=2 \bar{e}, \bar{x}=-1 \ldots 0 \ldots$.

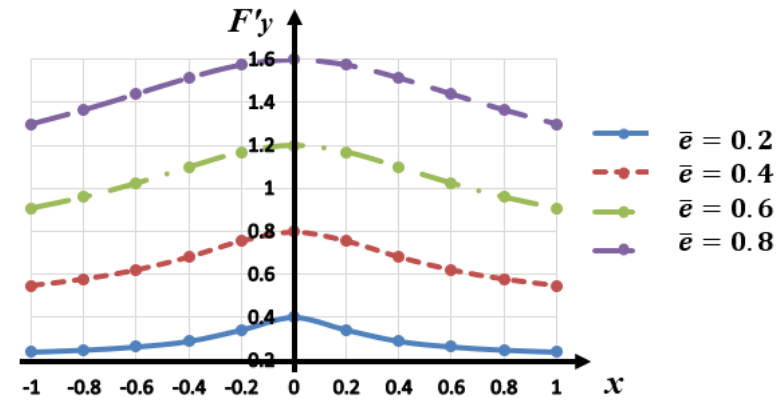

Fig. 7. Graphs of $\bar{F}_{y}^{x}$ along the guides with $\bar{s}_{f}=2 \bar{e}$.

When $\bar{e}=\bar{s}_{\mathfrak{l}}$, the load $F y$ along the guides is constant and equal to:

$$
E_{y}=c \cdot \bar{e} \cdot x_{\max } .
$$

With a single stiffness $c=1$ and $F_{y}=e \cdot \bar{s}_{\perp}=\bar{e}, F=c x$ and $t=\sqrt{e}$. Obviously, with an increase in the preliminary spring force, we can obtain an even smaller value of the coefficient $K_{0}$.

To compensate for dissipative losses, it is necessary to know the energy losses in the elements of the spring accumulator. Although the losses in the moving elements of the spring accumulator are determined by design features, the most common for any versions are identified in the work: losses on internal friction in springs, losses in translational pairs and in hinge joints.

Dissipative losses are primarily determined by the configuration of the nonlinear spring battery and its two main dimensionless characteristics $\bar{e}$ and $\bar{s}_{\mathbb{l}}$.

The work on compensation of dissipative losses on internal friction in springs $A_{d l}$ is determined from the expression:

$$
A_{\text {dI }}=\varphi U b \text {, }
$$

where, $\varphi$ - is the coefficient of losses in the spring on internal friction, it can be taken equal to $\varphi=0.1$.

The work on compensation of dissipative losses in the guides from the weight of the carriage $A_{d 2}$ is determined from the expression:

$$
A_{d 2}=2 \cdot m g f x_{\max },
$$

where, $f-$ is the coefficient of sliding friction or the reduced coefficient of friction when using rolling guides.

The work to compensate for the dissipative losses in the carriage guides from the spring force $A_{d 3}$ is determined by the following equation:

$$
A_{d 3}=\frac{2 m x^{2}-f}{t^{2}} \cdot K_{3}
$$

Where $K_{g}$ - coefficient of losses from friction in the carriage is determined by the force of the spring.

$$
K_{g}=K_{\theta}^{2} \bar{\theta} \cdot\left[1-\left(\bar{e}-\bar{s}_{p}\right) \cdot \ln \frac{1+\sqrt{1+\epsilon^{2}}}{\epsilon}\right],
$$


The work to compensate for dissipative losses in $A_{d 4}$ hinged joints, in accordance with [18], is defined by the following expression:

$$
A_{d 4}=\frac{2 m x^{2} \cdot f}{t^{2}} \cdot K_{l}^{2}
$$

where,

$$
\begin{gathered}
K_{1}=\left[\ln \frac{1+\sqrt{1+\bar{e}^{2}}}{\bar{e}}-\left(\bar{e}-\bar{s}_{1}\right) \cdot \operatorname{arctg} \frac{1}{\bar{e}}\right] \\
d \cdot K_{\theta}{ }^{2} \cdot \bar{\theta},
\end{gathered}
$$

and $d$ - diameter of the hinge axis.

Fig. 8. shows the graphs of the change of the coefficients $K_{l}$ to determine dissipative losses in hinge joints depending on $\bar{e}$ and $\bar{s}_{\mathfrak{l}}$.

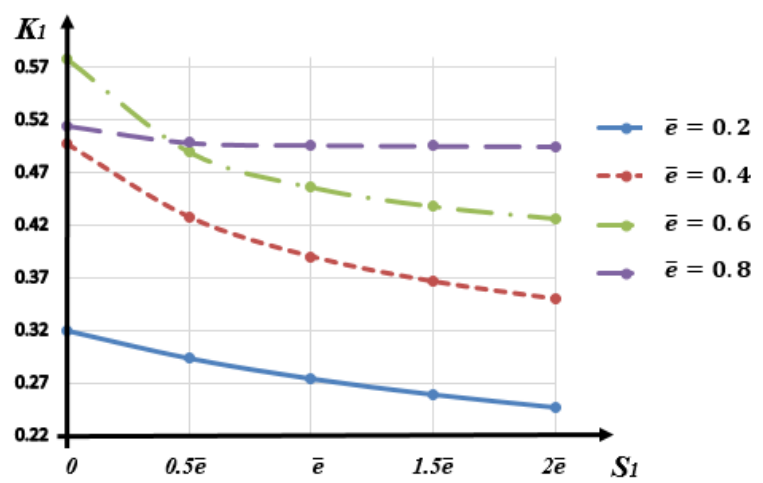

Fig. 8. Graphs of changes in coefficients $K_{l}$ depending on $\bar{\theta}$ and $\overline{s_{p}}$.

The work on compensation of dissipative losses in the rocker stone $A_{d 5}$ is defined by the following expression:

$$
A_{d 5}=\frac{2 m x^{2} \cdot f^{2}}{t^{2}} \cdot K_{2}^{2}
$$

where,

$$
K_{2}=\frac{d}{\theta} \bar{\theta}\left[1-\left(\bar{\theta}-\bar{s}_{1}\right) \cdot \ln \frac{1+\sqrt{1+\varepsilon^{2}}}{\epsilon}\right] \cdot K_{0}{ }^{2},
$$

Fig. 9 shows the graphs of changes in the coefficients of loss of $K_{3}$ friction in the carriage of the spring force and the loss coefficients of $K_{2}$ in the carriage of the spring force depending on $\bar{e}$ and $\bar{s}_{\mathbb{l}}$.

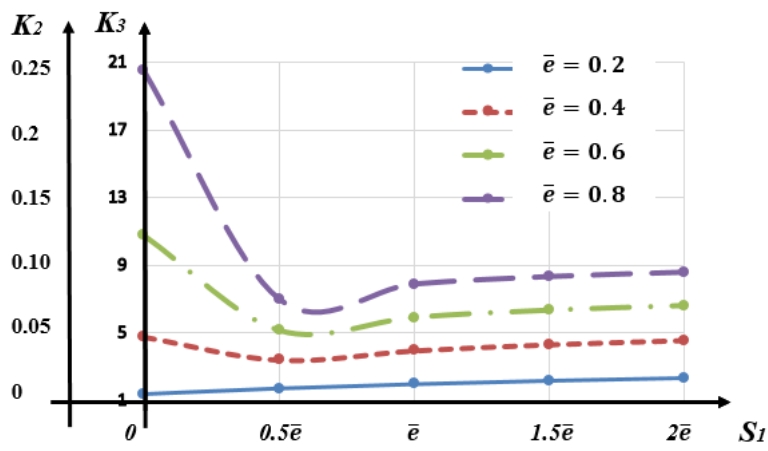

Fig. 9. Graphs of changes in coefficients $K_{2}$ and $K_{3}$ depending on $\bar{\varepsilon}$ and $\bar{s}_{g}$.

\section{Analysis of the Results}

Analysis of the equations shows that in these equations there are components that depend only on the geometric parameters $e$ and $s_{1}$ and the design parameters $f$ and $d$. Indeed, the coefficient of friction is determined by the choice of structures and materials of moving elements. The sizes of the diameters of the diameters of the axles of the hinged joints with the selected mass range lie in the range from 8 to $50 \mathrm{~mm}$ or $0.008-0.05 \mathrm{~m}$, for quantitative estimates, the diameter of the axis was taken to be $0.03 \mathrm{~m}$, the size $l$ was taken equal to $5 d$.

Since the friction losses from the carriage mass with a unit friction coefficient are determined by the acceleration of gravity and the friction coefficients, by taking the friction coefficients in all moving elements equal, the dissipative loss coefficients can be compared with the value $g=9.81$.

\section{Conclusion}

1. The coefficients of dissipative losses in the joints and rocker stone are more than 10 times less than the losses in the carriage.

2. The coefficients of losses in the carriage from the spring force are commensurate with the losses in the carriage from the mass of the moved load or product. Friction losses in the guides are reduced if the springs are located in a vertical plane and above the guides. In the particular case, when the condition $\mathrm{e}=\mathrm{mg} / \mathrm{c}$ is fulfilled, the load from the moved mass is balanced by the vertical component of the spring force throughout the entire working displacement [12]. At the same time, the energy costs of the engines to compensate for dissipative losses are reduced.

3. With a symmetric arrangement of the springs in the horizontal plane in the spring drive, the dissipative losses are determined only by the friction forces in the guides, from the mass moved in the horizontal plane, and from the friction forces in the hinged joints and in the rocker stone.

When $s_{1}=2 e$, it is possible to increase the mass of the load by 2 times, while maintaining the strength characteristics.

4. In comparison with electric, pneumatic and hydraulic drives, the energy costs for working operations for moving products can be reduced by more than 5 times.

5. From the point of view of dynamic loads, the most favorable are two ways to compensate for dissipative losses:

- a preliminary increase of the potential energy of the spring accumulator;

- compensation of dissipative losses throughout the carriage movement;

- each of these methods has limitations: in the first case it is the dwell time and the engine power to compensate for dissipative losses, in the second case it is the required characteristics of the drive in terms of speed. 
6. For fixing the carriage in extreme positions, for example, pneumatic cylinders with clamps can be recommended, and for working with large masses, hydraulic cylinders can be used if they provide linear velocities of the order of $1 \mathrm{~m} / \mathrm{s}$.

The developed drive for pulling the packing tape using a spring-loaded pneumatic cylinder reduces energy costs and provides favorable dynamic operating conditions. The results of the work were used in the development of the mechatronic pneumatic system of the drives of the filling and packaging machine for bulk products in the range from $0.5 \mathrm{~g}$ to $5 \mathrm{~g}$. The results of the work can benefit from the developers of energy-saving technological equipment.

\section{References}

1. V. I. Bezhanov, B. N. Bushunov, Production automatic machines, Mechanical Engineering, 360, (1973)

2. V. N. Pipunyrov, The history of watches from ancient times to the present day, The science, 496, (1982)

3. A. A. Andronov, A. A. Vitt, S. E. Khaikin, Oscillation theory, The science, 918, (1981)

4. G. Kellhoff La formule $\mathrm{d}^{\wedge}$ Airy.-Mem. Acad. Bel., 1897/ Vol 5 №11

5. N. N. Bautin, Dynamic clock theory, The science, 192, (1986)

6. Patentschrift №562040 Dr. Leo Szilard und Dr. Albert Einstein. Elertromagnetische Vorrichtung zur Erzeugung einer oszillierenden Bewegungen. 1933 Prioritatsdatum 1.Juni 1928.

7. V. Ya. Frenkel, B. E. Yavelov, Einstein inventor, The science, 160, (1982)

8. G. A. H. Roderstram, Mechanical hand, Patent №568346 (the USSR), (1974)

9. V. V. Zaitsev, A. I. Karandashev, A. P. Litovchenko, B. L. Salamandra, L. I. Tives, V. S. Shishkov, L. B. Sachkov, Industrial robot, Patent UR №1768381, (1983)

10. S. N. Sysoev, A. A. Glushkov, Oscillating cyclic drives, Feder. Education Agency, Vladimir. state unt, 184, (2010)

11. I. V. Nadezhdin, Highly dynamic mechanisms of auxiliary operations of automated assembly plants, Engineering, 270, (2008)

12. V.L. Zhavner, O.N. Matsko, Spring drives for reciprocal motion. Journal of Machinery Manufacture and Reliability, №1, 1-5, (2016)

13. V. I. Babitsky, A. A. Kulakov, V. A. Chekurov, A. V. Shipilov, V. N. Panin, Resonant drive, Patent №1544550 (the USSR), (1990)

14. A. Et. Levin, Mathematical modeling in research and design of machine tools, Engineering, 184, (1978)

15. L. D. Landau, E. M. Lifshits, Theoretical physics, The science, 216, (1988)
16. A. I. Korendyasev, B. L. Salamandra, L. I. Tives, Theoretical foundations of robotics, The science, 376, (2006)

17. A. I. Korendyasev, B. L. Salamandra, L. I. Tives, V. L. Zhavner, Robot handling systems, Engineering, 279-286, (1989)

18. D. S. Pelupessy, M. V. Zauner, Spring accumulators with an output rotary link for stepping movements, Proceedings of higher educational institutions. Engineering, № 10, 9-17, (2016)

19. V. L. Zhavner, O. N. Matsko, M. V. Zhavner, Comparative Analysis of Mechatronic Driver for Reciprocal Motion, I.RE.M.E., № 9, 784-789, (2018) 